\title{
Engage Decision Makers or Someone Else Will: The Need for More Compelling I-O Psychology Communication
}

\author{
Marc Sokol \\ Sage Consulting Resources, LLC
}

Better focus on the frontier of talent management (TM), the thesis of the focal article by Rotolo et al. (2018), is necessary but not by itself a sufficient condition for I-O psychologists (IOP) to influence organizational decision makers. Although I agree with the substance of their perspective, as professional psychologists we also need to communicate in a manner that decision makers recognize as worthy of their time and attention. We should pay greater attention to how, why, and when organizational stakeholders embrace, reject, or overlook our insights and recommendations.

Rotolo and colleagues open up much needed discourse on the evolution of our professional work. The argument of industrial-organizational psychology vs. anti-industrial-organizational psychology (AIO) strikes an emotional chord, much as we experience in the broader assault on science in some aspects of popular media. Compelling as this is, the authors seem to be also caught in a polarized view of good versus bad science, almost as if there are good versus evil actors within the field (Hambrick \& Marquardt, 2018; Johnson, 2014). Even with great research, however, we do not consistently see traction in terms of receptivity and application, and wonder how others without our foundation make headway. One opportunity is to pay more attention to how we communicate our message.

There is much we can do to better engage decision makers and influencers so that our messages are noticed and to create a desire for real dialogue about what we have learned. Given the flood of information that appears in every format, from social media to print options, IOP must be willing and ready to proactively compete for attention so that the quality of our professional work gains the traction we seek.

\section{Empathy for Consumers of TM-Related Communication}

The perspective begins with a narrative to illustrate the issue of AIO messaging getting to decision makers, well before the in-house I-O psychology professional is called in. Although we can sympathize with the

Marc Sokol, Sage Consulting Resources, LLC.

Correspondence concerning this article should be addressed to Marc Sokol, Sage Consulting Resources, LLC, 6417 Timber Ridge, Edina, MN 55439. E-mail: marc.sokol@SageHRD.com 
professional's predicament, we might also wonder how and why such events come about.

My experience is that we have done a much better job communicating to and for each other than to and for the other audiences we hope to inspire. Society for Industrial and Organizational Psychology (SIOP) has made strides to increase the volume and range of IOP practitioner-focused outlets, but what of our communication aimed toward organizational leaders and decision makers?

The growth of popular business books, blogs, and white papers continue, accompanied by an expanding array of TED talks. Ironically the more books, the more blogs, the more dramatic the headlines, the more we seem to inoculate leaders from attending to the onslaught of so much messaging. Many organizational leaders, including CHROs, claim they have little time to read unless they can see direct application and the material comes from a trusted source.

At other times a well-marketed book will capture attention of a key stakeholder, and this entry point drives broader reception throughout the organization. I have seen the president of one firm ask his high potentials to read Daniel Kahneman's (2011) book Thinking, Fast and Slow, and to apply that to how they think about that business. Similarly, leaders in financial services have begun to incorporate aspects of behavioral economics into their own presentations to large teams. So these leaders will read; our challenge is how to increase their likelihood of reading our work.

\section{Toward More Leader-Friendly Communication}

High potential programs, coaches, and speakers at business offsite often present a compelling TM story. A book on the topic may be a take-away; an attendee may purchase a set for his or her team to read. Theories of talent management embedded in powerful narrative may be all a key influencer needs to become open to a "new and better way." To fully leverage best practices of our research, we also need to present messages that gain the attention of gatekeepers and influencers.

Between some thinly disguised sales efforts labeled as white papers and research publications too densely written for business leaders, there is a gap and we are not gaining attention to our work. However, there is the opportunity to provide brief, informative, readable material that is relevant to the line executive and CHRO, and with a clear eye toward stimulating dialogue and application. Harvard Business Review has long been the gold standard but does not rest alone.

People + Strategy is the professional publication of the HR People + Strategy Association (www.HRPS.org) and part of the Society of Human Resource Management (SHRM). During the past 2 years, while serving as 
executive editor, along with a diverse editorial board, we have focused on the following:

- Design the publication with a look and length suitable for executive audience. Articles three to five pages in length, each typically no more than a 10-15 minute read.

- Digestible research findings and summaries, often using call out boxes to highlight key messages and infographics style tables or figures.

- Topical issues intended to reflect the role of HR and TM in some broader theme that executives likely resonate with: enterprise security, candor and transparency, maximizing potential, and so on.

- Embrace a variety of voices and styles of presentation: executive roundtables, first person accounts of from thought leaders and senior executives, case studies, and a regular column summarizing published research for its practice implications.

In the past several years, association membership has more than doubled, and representation of CHROs has increased from 12\% to 35\% (personal communication, L. Connell, March 7, 2018). During this time, we have reached out to IOPs and many have contributed to the journal. As an illustration, I provide links to two issues of People + Strategy, published in 2016. The first took on a broader topic of enterprise security as an organizing theme (http://www.nxtbook.com/ygsreprints/HRPS/hrps_39_1_2016/). IOPs contributed articles on cybersecurity (Klimoski, 2016), safety culture (Nieminen, Bianco, \& Denison, 2016), active shooter response on a college campus (Baran, 2016), and prediction of accident-prone tendencies (Hogan, 2016). Although it is 2 years since the publication date, every one of these topics is still highly relevant.

A second issue, devoted to the theme of candor and transparency in the workplace, also contained contributions from members of our profession (http://www.nxtbook.com/ygsreprints/HRPS/hrps_39_4_2016/). One contribution by two authors of the focal article (Church and Rotolo, 2016), in particular, reports research on the question of whether one should inform employees of their assessed potential. This article can stimulate dialogue among almost all TM Centers of Excellence and set the stage for similar discussion between $\mathrm{HR}$ and the $\mathrm{C}$-suite. This article, in fact, worked its way up to the executive committee of the author's company, a Fortune 50 firm (personal communication with Allan Church, March 31, 2017). Imagine if this experience was typical rather than the exception! Executives may always approach their TM leaders to act on "hot" ideas they have heard about, but I suspect that where IOP professionals have been actively communicating best practices, there is less likelihood of outside influence gaining traction without the support of in-house professionals. 
I can imagine an alternative narrative to follow the opening one of the focal article-one in which the CHRO had become used to the IOP professional providing brief, relevant, compelling articles on TM practices, and that routinely created a pull for discussion with members of the $\mathrm{C}$-suite. When approached by the external firm in the opening narrative, the CHRO countered its advances due to its lack of published results the CHRO could share with line leaders.

\section{Recommended Actions}

1. Editorial board members of People + Strategy who are affiliated with business schools have for several years joined with representatives of Harvard Business Review to host a panel at Academy of Management (AOM) on the topic of publishing for business leaders (Winn et al., 2015; Winn et al., 2016; Winn et al., 2017). This may seem atypical for AOM, but some professionals are making a concerted effort to convey a message about more impactful communication to executives. SIOP, through its annual conference, might host more sessions on communication of our research and best practices for non-IOP audiences. We might even devote an hour on the topic at doctoral consortia meetings. Notable on the 2018 conference schedule is a preconference workshop on storytelling to increase influence (Sinar \& Grubb, 2018) and a community of interest session on research about communicating with organizational leaders (Lin, Nolan, \& Dalal, 2018).

2. Although we often talk about bridging the scientist-practitioner divide, one way might be collaboration in publishing summaries of research that highlights application in formats that senior leaders are more likely to consume. With so many IOP practitioners based internally or working extensively inside organizations, we should leverage these materials to initiate and shape dialogue with organizational decision makers.

3. Graduate programs at the master's and doctoral level might add consideration of as well as training and practice in how we present our insights and findings to a wide range of stakeholders.

Rotolo et al. (2018), like most readers of Perspectives, want IOP to be a driving force in talent management theory and practice. Expanded strategies and tactics of communicating what we know will help further these aspirations.

\section{References}

Baran, B. (2016). High-reliability HR: Preparing the enterprise for catastrophes. People + Strategy, 39(1), 34-38. 
Church, A., \& Rotolo, C. (2016). Lifting the veil: What happens when you are transparent with people about their future potential? People + Strategy, 39(4), 36-40.

Hambrick, D., \& Marquardt, M. (2018, February 6). Cognitive ability and vulnerability to fake news. Scientific American. Retrieved from https://www.scientificamerican.com/article/ cognitive-ability-and-vulnerability-to-fake-news/.

Hogan, R. (2016). The accident-prone personality. People + Strategy, 39(1), 20-23.

Johnson, B. (2014). Polarity management: Identifying and managing unsolvable problems. Amherst, MA: HRD Press, Inc.

Kahneman, D. (2011). Thinking, fast and slow. New York, NY: Farrar, Strauss, and Giroux.

Klimoski, R. (2016). Critical success factors for cybersecurity leaders: Not just technical competence. People + Strategy, 39(1), 14-18.

Lin, L, Nolan, K., \& Dalal, D. (2018, April). Communicating with organizational leaders: Selling our intervention. Community of Interest presented at the 33rd Annual Conference of the Society for Industrial and Organizational Psychology, Chicago, IL.

Nieminen, L., Bianco, C., \& Denison, D. (2016). Transforming the safety culture at New YorkCity Transit. People + Strategy, 39(1), 24-28.

Rotolo, C. T., Church, A. H., Adler, S., Smither, J. W., Colquitt, A. L., Shull, A. C., ... Foster, G. (2018). Putting an end to bad talent management: A call to action for the field of I-O -psychology. Industrial and Organizational Psychology: Perspectives on Science and Practice, 11(2), 176-219.

Sinar, E., and Grubb, A. (2018, April). Storytelling with impact: Mastering the practical science of influential communication. Workshop presented at the 33rd Annual Conference of the Society for Industrial and Organizational Psychology, Chicago, IL.

Winn, B, Bernstein, E., Harrell, E., Mathieu, J., Luciano, M., Rogers, K., \& Tavis, A. (2017). Publishing at the research-practice interface: Editors \& scholars share tips for real world impact. Paper presented at the 77th Meeting of the Academy of Management: Annual Conference, Atlanta, GA.

Winn, B., Luthans, F., Hayton, J., Tavis, A., Abel, A., \& Merino, M. (2015). Publishing beyond the Academy: Editors share tips on publishing for impact in the "real world". Paper presented at the 75th Meeting of the Academy of Management: Annual Conference, Philadelphia, PA.

Winn, B., Ulrich, D., Rucci, A., Bernstein, E., Merio, M., \& Tavis, A. (2016). Publishing for real world impact: Editors and researchers share tips on making research meaningful. Paper presented at the 76th Meeting of the Academy of Management: Annual Conference, Anaheim, CA.

\title{
Forever New Frontiers: Managing Messiness at the Edge
}

\author{
Paul R. Yost and Matthew S. Magill
}

Seattle Pacific University

A life's work is not a series of stepping-stones onto which we calmly place our feet, but more like an ocean crossing where there is no path, only a heading, a direction, which, of itself, is in conversation with the elements.

David Whyte, Crossing the Unknown Sea

Paul R. Yost, Seattle Pacific University; Matthew S. Magill, Seattle Pacific University.

Address correspondence to Paul R. Yost, Seattle Pacific University, Department of Industrial-Organizational Psychology, 3307 Third Avenue West, Suite 107, Seattle, WA 981191922. E-mail: yostp@spu.edu 